\title{
Reconfigurable Virtual Environment for Multi- robot Operations and its Application in Education
}

\author{
Maja M. Lutovac, Zoran Dimić, Stefan Mitrović, Aleksandar Stepanović, and Vladimir Kvrgić
}

\begin{abstract}
This paper presents the development of reconfigurable virtual environment for programming, control, simulation, and monitoring of multiple robots and its application in the process of education. During the development special attention is devoted to the fulfillment of the modularity, scalability, and openness that represent the basic requirements of reconfigurability. The reconfigurability of the virtual laboratory can be considered from two aspects: as a possibility of reconfiguring operating mode and as a possibility of reconfiguring virtual robots and virtual laboratory. It represents an integrated environment for programming, control, simulation and monitoring of multiple robots with continuous adjustment to the real laboratory environment and robots' usage. With the possibility to adjust the virtual environment in accordance with the students' foreknowledge and experience by combining different operating modes and virtual environments, from the educational viewpoint, it is expected that the proposed solution will improve the process of gaining knowledge.
\end{abstract}

Keywords - Education, Reconfigurability, Robot programming, Virtual laboratory.

\section{INTRODUCTION}

$\mathrm{W}$ ITHIN the disciplines of engineering sciences, laboratory work is considered to be "at the heart" of learning and can have a strong impact on students' learning outcomes. Also, sessions based on the use of laboratories are widely used in order to provide physical

Paper received May 9, 2016; revised July 14, 2016; accepted August 6, 2016. Date of publication November 20, 2016. The associate editor coordinating the review of this manuscript and approving it for publication was Prof. Jovan Đorđević.

This paper is a revised and expanded version of the paper presented at the 23rd Telecommunications Forum TELFOR 2015 [25].

This research was performed as part of a research project supported by the Ministry of Education and Science, Republic of Serbia, under grant TR35023.

Maja M. Lutovac, School of Electrical Engineering, University of Belgrade, Bul. kralja Aleksandra 73, 11120 Belgrade; Lola Institut, Kneza Višeslava 70a, 11130 Belgrade, Serbia (phone: 381-63-8639871; e mail: maja.lutovac@li.rs),

Zoran Dimić, Lola Institut, Kneza Višeslava 70a, 11130 Belgrade, Serbia (e mail: zoran.dimić@li.rs).

Stefan Mitrović, Lola Institut, Kneza Višeslava 70a, 11130 Belgrade, Serbia (e mail: stefan.mitrovic@li.rs).

Aleksandar Stepanović, Lola Institut, Kneza Višeslava 70a, 11130 Belgrade, Serbia (e mail: aleksandar.stepanovic@li.rs).

Vladimir Kvrgić, Lola Institut, Kneza Višeslava 70a, 11130 Belgrade, Serbia (e mail: vladimir.kvrgic@li.rs). evidence of theoretical principles and to teach practical skills. Nevertheless, laboratory teaching requires commitments of time, space, and finance to purchase, install, and maintain equipment [1].

Nowadays, virtual and/or remote laboratories have become the "must-have" in the modern, global, and successful teaching environment [2]. The increasing need of such environments in robotics is reflected in the existence of a large number of software systems and environments for robotics education [3-9]. A Virtual Laboratory for Robotics (VLR) was developed at the School of Electrical Engineering, University of Belgrade [3]. In order to give students an accurate impression of working with real equipment, the VLR involves simulation models to emulate system dynamics, user friendly interface and advanced graphics to provide visualization. It can be used for experiments and exercises in training future robot operators, but also for helping students to learn robotic theory. With the aim to introduce the concepts of robotics and artificial intelligence (AI) to young students, a $3 \mathrm{D}$ robot simulation for education is proposed in [4]. It allows students to create and manipulate their own AI, giving unlimited access to a realistic robotic simulation. An interactive educational tool for cognitive robotic education (ReAct!) is presented in [5]. ReAct! helps students to model a dynamic domain in a logic based formalism and solve a hybrid planning problem. Reference [6] describes Pyro - Python-based robotics programming environment. Its goal is to reduce the cost of learning to program robots by creating uniform conceptualizations that are independent of specific robot platforms and incorporate them into an already familiar programming paradigm. Reference [7] describes a tool for robotics education named Urban Search and Rescue Simulation (USARSim). It is a general purpose multirobot open source simulator. A Distance Laboratory System (DLS) that enables remote testing of control algorithms in robotic manipulators is presented in [8]. The DLS helps students to understand how the controller's design affects the robot manipulator performance and to deepen their study of robot manipulator kinematics and dynamic models. A more detailed review of existing virtual and remote laboratories in the field of robotics can be found in [9].

Virtual robot laboratories are based on software to simulate experiments environment, while in remote 
laboratories experiments are conducted and controlled remotely through Internet, using real robots or devices [10]. Reference [3] notes the advantages and advanced features of a virtual laboratory over a real one: massive reconfiguration and extreme tasks are possible, a view inside the robot cover is allowed, etc., and points out the well-known benefits associated to the implementation of simulators in a training process: cost efficiency, accessibility, free schedule, energy saving, time saving for both students and staff, lack of damage to robots and the environment, no break-downs, no necessity for reserve expendable items, etc. Although the remote-access-to-reallab concept is completely different from the simulationmodel-based concept, the two concepts are not in conflict [3]. Remote laboratories give real-life feedback to the student and offer a more realistic and dynamic perspective [2]. So it is important to have both options: the virtual and the remote laboratory.

Reconfigurability of production systems facilitates cost effective and rapid responses to market and product changes [11]. Reconfigurability can be seen as a possibility to reconfigure any of the production segment, machine tool, robot, or multi-robot system. Considering the fact that contemporary machine tools and robots are computer controlled machines, reconfigurability must be accompanied by configuration changes in the computer hardware and software. The ability to configure and reconfigure control system, according to the literature [12] depends on the openness, modularity and scalability of the control architecture. To make the control system applicable in emulation of multi-axis machine tools on industrial robots, the corresponding software control modules, such as kinematic, interface modules, etc., must be available for modification and/or extension. This implies the openness of the control software code and the availability of adequate compiler tools. The reconfiguration of control architecture must be accompanied by appropriate tests and simulations to verify the functionality and safety of the newly created configuration. Because of that, the configuration interface and the corresponding verification tools must be an integral part of the reconfigurable control system.

Robot simulation is very important in complex reconfigurable robotic environment and modern industrial applications [13-18]. There is much current research on robotic system simulation. Industrial robot manufacturers provide commercial software for offline programming simulation, such as ROBCAD and IGRIP, which include robot simulation tools $[19,20]$. Considering the fact that available commercial systems do not have an open architecture, according to the literature [12] they cannot have all the features of reconfigurability.

The paper proposes the reconfigurable virtual multirobot laboratory as a stand-alone software application, but which can be configured as an integral part of a robot programming and control system. During the research and development of the proposed system, special attention was devoted to the fulfillment of the basic requirements of reconfigurability: modularity and scalability. The system was developed by using open architecture software tools, what is in line with the established requirements.

The virtual environment enables verification of multirobot program that includes the following:

- Check if the robot can execute the specified path within the limited joints motion ranges and speeds;

- Visual detection of collisions between the robots and the tools with surrounding environment.

The reconfigurability of the virtual robot laboratory can be considered from two aspects:

- as a possibility of reconfiguring operating mode;

- as a possibility of reconfiguring virtual environment.

The paper describes a reconfigurable virtual multi-robot environment which can be directly used for robot programs testing, simulation and execution. The proposed reconfigurable laboratory can operate:

- as a virtual laboratory, by using a simulation mode, and

- as a remote laboratory, by using remote wireless communication with the real laboratory.

Because of its ability to adapt in accordance with the students' foreknowledge and experience by combining different operating mode and virtual environments, the proposed solution can be suitable for application in education, in the robotics field. Virtual laboratory can be used to introduce less experienced students to the programming and control of robots using simulations. Remote laboratory can be used to teach students with a strong mathematics and programming background how to program robot control programs and to help them to gain an insight into the execution of such programs by using remote access to the robotic laboratory, since the real laboratory is usually inaccessible for them.

The laboratory can be reconfigured from the simple, one-robot environment to the complex, multi-robot environment. It also includes the possibility of operating mode reconfiguration. This kind of reconfigurable laboratory is suitable for adapting the virtual environment to be in accordance with the current students needs. The paper describes the application of the proposed reconfigurable environment for educational purposes, for two different operating modes, at practical classes that took place at the Lola Institute. In the first stage of application of this reconfigurable environment, the programming of robot programs is performed within the L-IRL programming environment by using Lola Industrial Robot Language [21].

The rest of the paper is organized as follows. The design and implementation of the proposed environment is given in Section II. Description of the reconfigurable virtual environment for multi-robot operations is presented in Section III. The application of the proposed environment for the virtual laboratory configuration is outlined in Section IV. The application of reconfigurable virtual environment in the education process is described in Section V. The concluding remarks are given in Section VI. 


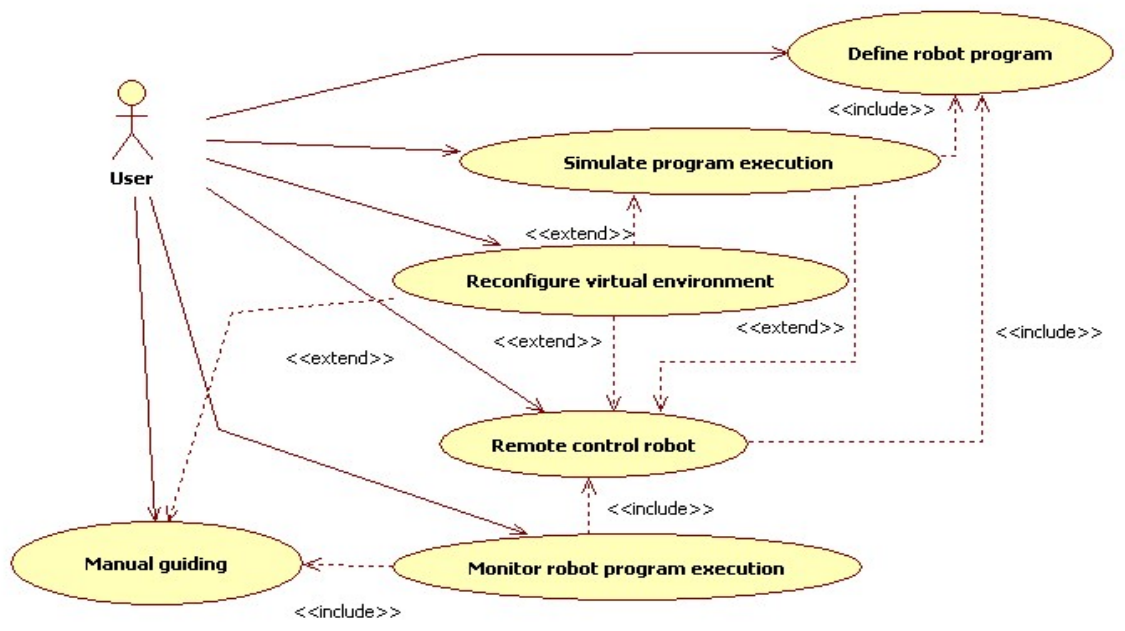

Fig. 1 Use-case diagram of the virtual environment for multi-robot control.

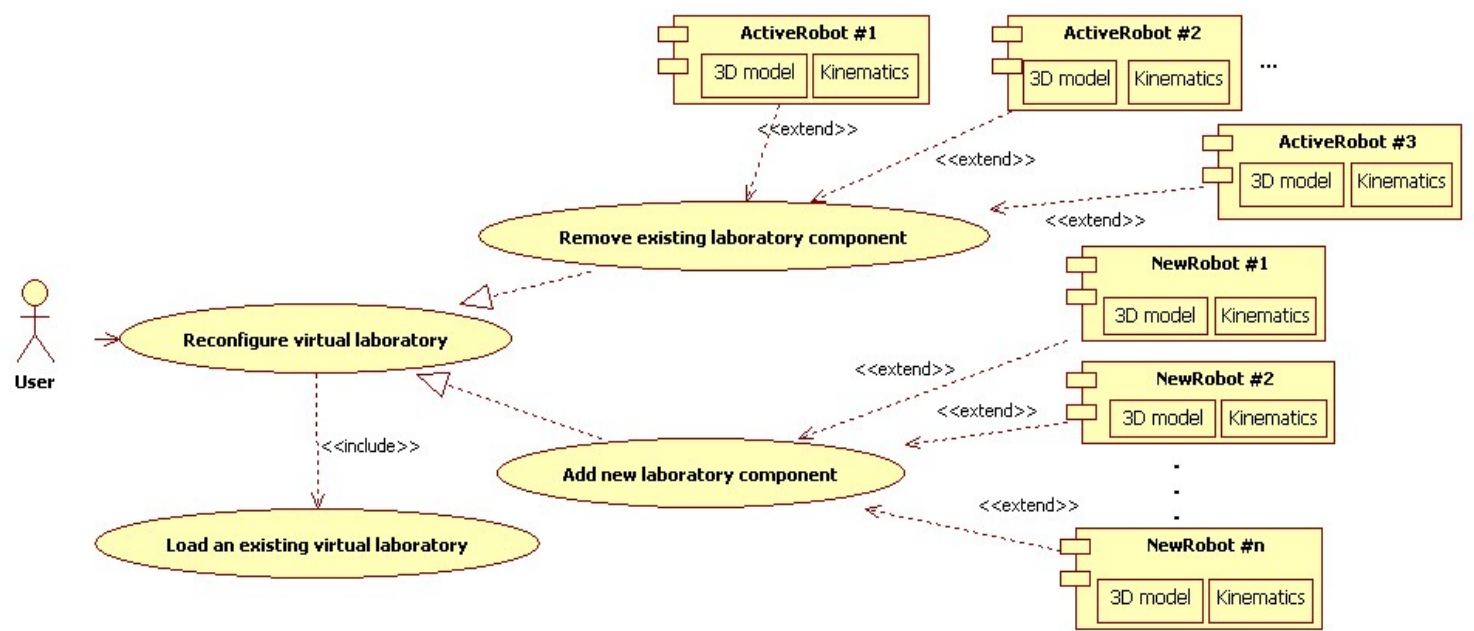

Fig. 2 Use-case diagram of the virtual laboratory reconfiguration.

\section{DESIGN AND IMPLEMENTATION}

The use-case diagram for the proposed environment is shown in Fig. 1. It depicts a high-level view of the system functionalities. Four main functionalities of the environment are:

- Configuration/reconfiguration of the virtual laboratory;

- Manual guiding;

- Simulation of the programmed multi-robot tasks using configured virtual laboratory model;

- Execution of programmed multi-robot tasks in a real laboratory and remote monitoring using $3 \mathrm{D}$ virtual environment.

Virtual environment can be adjusted to the current laboratory environment by configuring/reconfiguring the virtual 3D model, i.e., by defining the number and kind of robots, additional working axis and robots' position and orientation within the virtual space. Testing and verification of robot programs can be performed by simulation of such configured environment. Further, the verified robot tasks execution in the real robot laboratory can be remotely initiated and their execution can be monitored by using the virtual environment that is adjusted to the specific robot program. Manual guiding of robot axis with remote monitoring complements the process of robot motion testing and verification.

The environment is implemented on the Linux platform using the $\mathrm{C}++$ programming language. The $3 \mathrm{D}$ visualization is implemented using the OpenGL libraries. For the implementation of the graphical user interface, the Qt libraries were used.

Modularity is achieved by decomposing the environment into independent software modules, each providing well-defined functionalities specific to a particular operating mode and robot manipulator. The usecase diagram of the virtual laboratory reconfiguration is shown in Fig. 2. Independent software modules of robot manipulators, which are included in virtual environment and which contain implementation of 3D model and kinematics algorithm for a specific robot manipulator, are marked with ActiveRobot\#1 - \#n. With the possibility of adding a new element (NewRobot \#i) or removing an existing (ActiveRobot \#i), in order to adjust the virtual environment in accordance with the real laboratory 


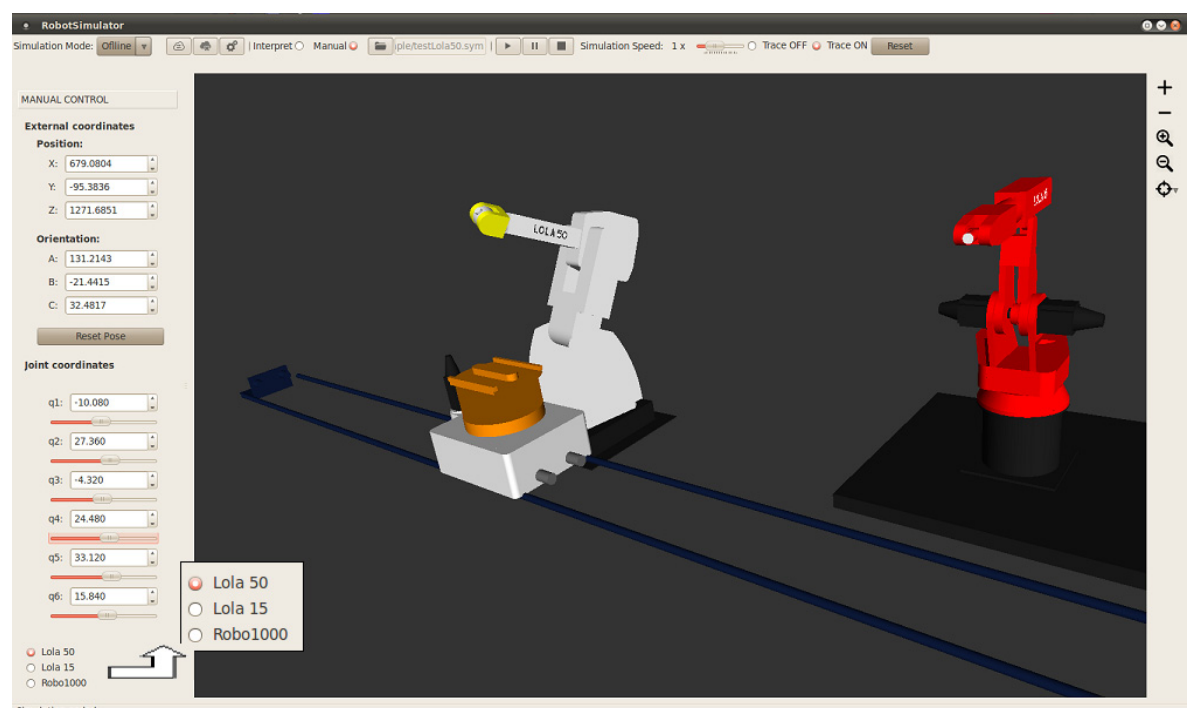

Fig. 3 The preview of the virtual environment for the configured multi-robot laboratory that operates in offline mode.

environment and robots' usage with a minimal effort, the scalability is accomplished. In order to provide openness, the environment was developed by using open architecture software tools.

Robots are programmed in a robot specific programming language that is developed at the Lola Institute - Lola Industrial Robot Language (L-IRL). LIRL compiler [21] translates those programs to the object code form that is sent to the real robot laboratory, by using CORBA protocol [22]. Those forms are also used for robot program simulation during the testing process. In

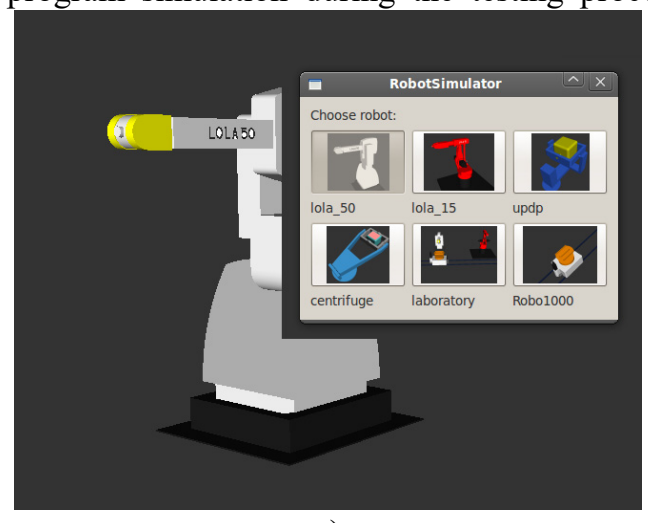

a)

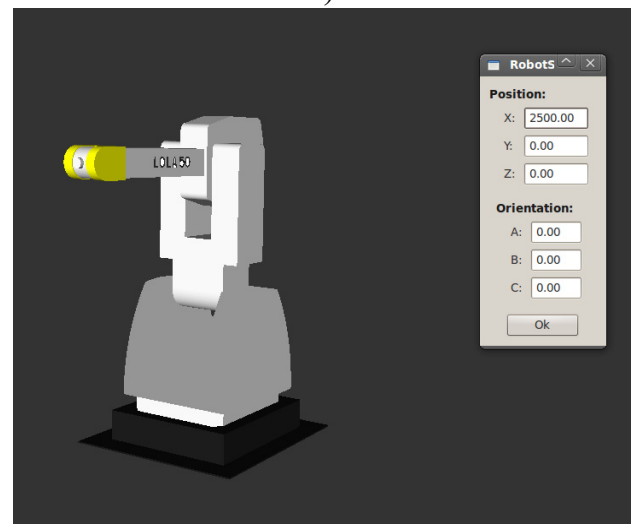

c) addition to the work that is described in [23] and [24] the paper describes a new version of the system which is reconfigurable. Reconfigurability is achieved with multiple operating modes and the possibility of configuring and reconfiguring multi-robot environment by including one or several robots, additional working axis and adjusting their position and orientation within the virtual space. Robot programs can be first programmed and tested on the individual robot, and then can be integrated into the multi-robot environment.

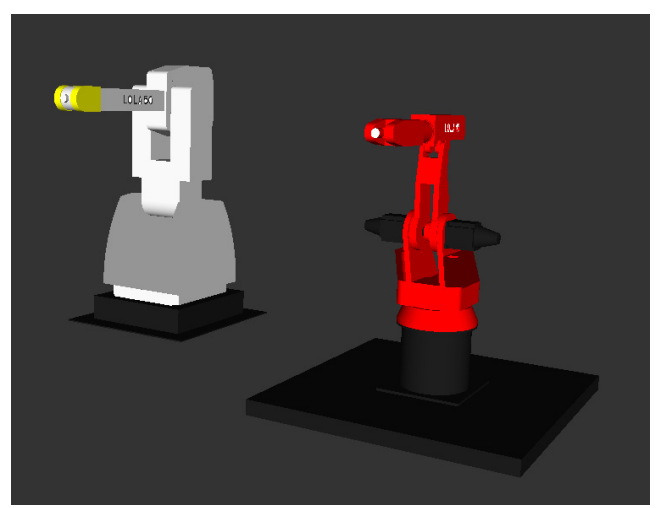

b)

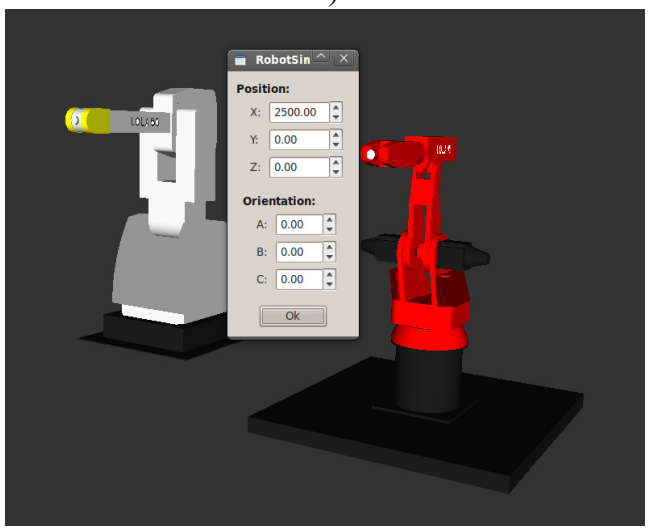

d)

Fig. 4 Graphical user interface of the virtual environment in the reconfigurable mode:

a) Robot model selection; b) New added robot's position and orientation setting;

c) Virtual laboratory with new added robot; d) Redefinition of robot's position and orientation. 


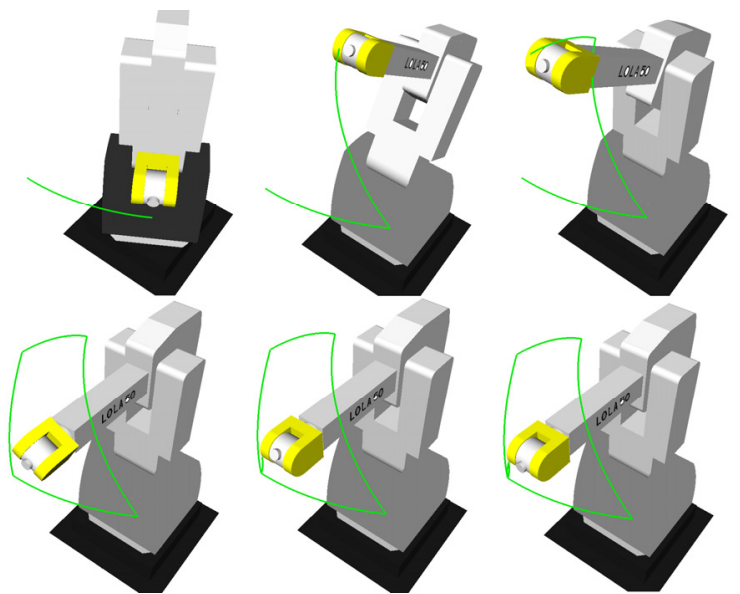

Fig. 5 Virtual robot Lola 50 and its end-effector's trajectory during the execution of the lesson in offline operating mode.

\section{RECONFIGURABLE VIRTUAL ENVIRONMENT FOR MulTi-RoBOt OPERATIONS}

The system includes four operating modes:

- Reconfigurable mode,

- Offline mode,

- Online mode,

- Manual mode.

The reconfigurable mode is used for defining and configuration of the virtual multi-robot environment in accordance with the real laboratory layout. The testing and verification of robot programs are conducted within the offline operating mode and the manual operating mode. If no errors are found during the simulation, the robot program is safe for implementation on the real robots. The online operating mode is used for remote real-time control of the real robot laboratory or the individual robot, and provides the preview of the tasks execution. The preview of the virtual environment for the configured multi-robot laboratory that operates in the offline mode is shown in Fig. 3. The number and kind of robots, additional working axis, and their position and orientation are defined within the reconfigurable operating mode. The virtual laboratory consists of two industrial robots (Lola 15 and Lola 50) and two additional working axes (that are defined with the one translational and one rotational axis of the robot Robo1000). The positions and orientations of robots are specified according to their positions and orientations in the real laboratory. The left part of the GUI shows inner and outer coordinates for the robot that is selected within the virtual laboratory (selection is performed on the leftbottom part of the GUI). After the verification of the specified program within the offline operating mode, it can be remotely sent to the real laboratory for execution.

\section{USING ViRTUAL ENVIRONMENT IN RECONFIGURABLE MODE}

The previews of the graphical user interface of the proposed environment for each step in reconfiguring the virtual laboratory, in the reconfigurable mode, is shown in Fig. 4. In the existing one-robot virtual laboratory with the industrial robot Lola 50, another industrial robot (Lola 15) is added. The new robot model is selected by using graphical user interface that is shown in Fig. 4 (a). The position of new added robot is set by using graphical user interface that is shown in Fig. 4 (b). The virtual laboratory with new added industrial robot Lola 15 is shown in Fig. 4 (c). Position and orientation for robots that are already included into the virtual laboratory can be modified to provide the possibility of adjustments to possible changes in real laboratory. The graphical user interface for existing robot's position and orientation adjustment within the virtual laboratory is shown in Fig. 4 (d).

\section{Application of the ReCONFIGURABle VirTuAL ENVIRONMENT IN EDUCATION}

A survey on usefulness of the proposed solution is conducted among electrical engineering students at practical classes that took place at the Lola Institute. The survey was conducted among 24 high school electrical engineering students. The application is carried out for two operating modes: the manual operating mode and the offline operating mode. At the first lesson, students use the proposed environment in the manual operating mode to perform the manual movement of each virtual axis of the industrial robot Lola 50. At the second lesson students use the proposed environment in the offline operating mode to perform the programming of the industrial robot Lola 50 motion by using the L-IRL programming language. Students are given an assignment to perform the movement of the industrial robot Lola 50 by using a separate PTP (Point to Point) instruction for the movement of each axis, and to verify that the robots can execute the specified paths within the limited joints motions ranges and speeds. The PTP instructions are defined within the LIRL language. Before performing this exercise, students were given an explanation and guidance on the use of LIRL PTP motion instructions and L-IRL programming environment.

The obtained results during the execution of one of the several successfully performed exercises of the second assignment are shown in Fig. 5. Fig. 5 shows positions of the virtual robot Lola 50 with the trajectory of its endeffector during the execution of robot program that consists of six PTP motions instructions. Each of PTP instructions moves one of the six robot axes for 30 degrees. The trajectory of robot's end-effector is marked with a green line within the 3D virtual space. Each one of the given six previews of Lola 50 depicts execution of a single PTP motion instruction with its end-effector's trajectory. Every upcoming PTP instruction is executed while its path is being drawn on the screen, altogether with the trajectories of previous instructions. After the completion of lessons, students are asked to give an opinion about the proposed solution. The Likert type scale ranging from " 1 ', indicating strong disagreement, to " 5 ', indicating strong agreement, was used to measure the participants' agreement level with the statements that concern the proposed solutions (simulation, remote access, learning complex robotics tasks). The general students' opinion was that the possibility of simulation and remote 
access to the robotic laboratory facilitates learning in robotics field (mean score 4.25) and is of great importance in the acquisition of practical knowledge when there is no possibility of regular use of robotic laboratory equipment (mean score 4.37). Students have found that the proposed environment is very useful for learning more complex robotics tasks in a gradual manner (mean score 3.91). Most students consider that the remote communication to the robotic laboratory can provide them an opportunity to advance knowledge in the robotics field that otherwise they would not be able to get due to the lack of equipment (mean score 4.67).

\section{CONCLUSION}

This paper presents a reconfigurable virtual environment for programming, simulation, and control of multi-robot operations. With the proposed design of the virtual multi-robot environment the abilities of openness, modularity and scalability are provided. Reconfigurability is achieved by introducing several operating modes, and the possibility of configuration of virtual laboratories according to the current laboratory layout and robots' usage. The result of application of this reconfigurable environment for educational purposes for two different operating modes, that took place at practical classes at the Lola Institute, and a survey on the usefulness of the proposed solution are described. With the use of reconfigurable laboratory with several operating modes and the possibility of adapting environment to the students' current knowledge and experience, this solution becomes suitable for use in education in the robotics field. The proposed environment can be extended by additional types of robots, by importing a 3D model and the appropriate kinematics dependencies for the new robot.

Further work on the development is oriented towards the self-reconfigurability of the proposed system that would be obtained by automatic data acquisition within the robotic laboratory and configuration of the virtual laboratory layout according to collected data.

\section{REFERENCES}

[1] B. Hanson, P. Culmer, J. Gallagher, K. Page, E. Read, A Weightman, M. Levesley, "ReLOAD: real laboratories operated at a distance," IEEE Transactions on Learning Technologies, vol. 2, no. 4, pp. 331-341, 2009.

[2] C. M. Ionescu, E. Fabregas, S. M Cristescu, S. Dormido, R. De Keyser," A remote laboratory as an innovative educational tool for practicing control engineering concepts," IEEE Transactions on Education, vol. 56, no. 4, pp. 436-442, 2013.

[3] V. Potkonjak, M. Vukobratović, K. Jovanović, M. Medenica, "Virtual Mechatronic/Robotic laboratory-A step further in distance learning," Computers \& Education, vol. 55, no. 2, pp. 465-475, 2010.

[4] T. L. Dunn, A. Wardhani, "A 3D robot simulation for education," in Proc. of the 1st international conference on Computer graphics and interactive techniques in Australasia and South East Asia, February 11 - 14, 2003, Melbourne, Victoria, Australia, pp. 277-278.

[5] Z. Dogmus, E. Erdem V. Patoglu, "REACT!: An interactive educational tool for AI planning for robotics," IEEE Transactions on Education, vol. 58, no. 1, pp. 15-24, 2015.

[6] D. Blank, D. Kumar, L. Meeden, H. Yanco, "Pyro: an integrated environment for robotics education," in Proc of the 20th national conference on Artificial intelligence, July 9-13, 2005, Pittsburgh, Pennsylvania, pp. 1718-1719.
[7] S. Carpin, M. Lewis, J. Wang, S. Balakirsky C. Scrapper, "USARSim: a robot simulator for research and education," in Proc of IEEE International Conference on Robotics and Automation, Apr. 10-14, 2007, Roma, Italy, pp. 1400-1405.

[8] A. R. Sartorius Castellanos, L. H. Santana, E. Rubio, I. Santana Ching R. A. Santonja, "Virtual and remote laboratory for robot manipulator control study," International Journal of Engineering Education,vol. 22, no. 4, pp. 702-710, 2006.

[9] V. Potkonjak, M. Gardner, V. Callaghan, P. Mattila, C. Guetl, V. M. Petrović, K. Jovanović, "Virtual laboratories for education in science, technology, and engineering: A review," Computers \& Education, vol. 95, pp. 309-327, 2016.

[10] L. Cui, F. P. Tso, D. Yao, W. Jia, “ WeFiLab: a web-based WiFi laboratory platform for wireless networking education," IEEE Transactions on Learning Technologies, vol. 5, no. 4, pp. 291-303, 2012.

[11] Y. Koren, M. Shpitalni, "Design of reconfigurable manufacturing systems," Journal of Manufacturing Systems, vol. 29, no. 4, pp. 130-141, Oct. 2010.

[12] Z. M. Bi, S. Y. T. Lang, M. Verner,P. Orban (2008) "Development of reconfigurable machines," International Journal of Advanced Manufacturing Technology, vol. 39, no. 11, pp. 1227-1251, 2008.

[13] J. DePree, C. Gesswein, Robotic machining white paper projectHalcyon Development, 2008. Available: http://www.robotics.org/robotic-content.cfm/Robotics/HalcyonDevelopment-RIA/id/43.

[14] W. Li, E. Red, G. Jensen, M. Evans, "Reconfigurable mechanisms for application control (RMAC): applications," Computer-Aided Design \& Applications, vol. 4, no. 1-4, pp.549-556, 2007.

[15] D. Milutinovic, M. Glavonjic, N. Slavkovic, Z. Dimic, S Zivanovic, B. Kokotovic, Lj. Tanovic, "Reconfigurable robotic machining system controlled and programmed in a machine tool manner," International Journal of Advanced Manufacturing Technology, vol 53, pp. 1217-1229, 2011.

[16] D. Milutinovic, N. Slavkovic, S. Zivanovic, M. Glavonjic, "Lowcost control and programming system for five-axis machining by articulated robots with 5 and 6 dof," in Proc. of 5th International Conference on Manufacturing Engineering ICMEN, Thessaloniki Greece, 2014, pp.133-142.

[17] S. Živanović, Z. Dimić, N. Slavković, D. Milutinović, M. Glavonjić, "Configuring of virtual robot for machining and application in off-line programming and education," in Proc. of 1 st International Scientific Conference Conference on Mechanical Engineering Technologies and Applications COMETa, Jahorina, B\&H, 2012, pp. 125-132.

[18] N. Slavkovic, D. Milutinovic, M. Glavonjic, "A method for off-line compensation of cutting force-induced errors in robotic machining by tool path modification," International Journal of Advanced Manufacturing Technology, vol. 70, 2014, pp. 2083-2096.

[19] C. S. Kim, K. S. Hong, Y. S. Han, "Welding robot applications in shipbuilding industry: Off-Line programming, virtual reality simulation, and open architecture," Industrial Robotics: Programming, Simulation and Application, LK Huat, Ed. ARS/pIV, 2006, pp. 537-558.

[20] Z. Pan, J. Polden, N. Larkin, S. van Duin, J. Norrish, "Recent progress on programming methods for industrial robots," Faculty of Engineering and Information Sciences - Papers, 2012, pp. 87-94.

[21] M. Lutovac, G. Ferenc, V. Kvrgić, J. Vidaković, Z. Dimić, "Robot programming system based on L-IRL programming language," Acta Technica Corviniensis - Bulletin of Engineering, vol. 2., 2012, pp. 27-30

[22] G. Ferenc, Z. Dimić, M. Lutovac, J. Vidaković, V. Kvrgić, “Open architecture platforms for the control of robotic systems and a proposed reference architecture model," Transactions of FAMENA, vol. 37, no. 1, 2013, pp. 89-100.

[23] M. Lutovac, Z. Dimić, G. Ferenc, J. Vidaković, M. Bućan, "Virtual robot in distributed control system," (in Serbian), in Proc. of 20. Telecommunications forum - TELFOR, Nov. 20-22, 2012, Belgrade, Serbia, pp. 1401-1404.

[24] S. Mitrović, Z. Dimić, J. Vidaković, M. Lutovac, V. Kvrgić, "System for simulation and supervision of robotic cells," in Proc. of 12th International Scientific Conference, Novi Sad, Serbia, Sept. 25-26, 2015.

[25] M. M. Lutovac, Z. Dimić, S. Mitrović and A. Stepanović, "Reconfigurable multi-robot virtual environment," Telecommunications Forum Telfor (TELFOR), 2015 23rd, Belgrade, 2015, pp. 954-957. 